\title{
Er, Cr:YSGG laser-assisted sinus lift surgery and implant placement. a case report
}

\begin{abstract}
Development of laser devices in a wide range of medical specialties is impressive. Numerous laser types have been applied in Oral and Maxillofacial surgery, with different indications and features.

Sinus lift elevation using bone grafts is a popular, scientifically documented method of bone augmentation, in cases with bone background of the area considered to be residual for implant placement.

A 47 years old patient was referred for a fixed implant-supported restoration on the upper left half of the maxilla. After clinical and radiographic evaluation, laser-assisted bone augmentation for open sinus lift technique, simultaneously with placement of endosseous implants was recommended.
\end{abstract}

Er, Cr: YSGG laser is a valuable tool in open sinus lift surgery, if used at recommended settings mode and doctor possesses appropriate medical training.

Keywords: laser-assisted surgery, Er, Cr, YSGG, Er, YAG, Nd, YAG
Volume 4 Issue 3 - 2016

\section{Titsinides S, Markopoulos George}

Department of Dentistry, National and Kapodistrian University of Athens, Greece

Correspondence: Titsinides Savvas, Department of Dentistry, National and Kapodistrian University of Athens, Greece, Tel +307000000000, Email titsinidess@yahoo.com

Received: February 04, 2016 | Published: February 26, 2016

\section{Introduction}

Laser devices, acronym for "Light Amplification by Stimulated Emission of Radiation", are widely applied in many fields of science nowadays. Development foundations were made by Albert Einstein, in 1916, by delivering the theory of "stimulated emission of radiation", which was implemented by Theodore Maiman constructing the first laser using Ruby crystal.

Practically, selection of a suitable type of laser is based on wavelength properties of beam, being absorbed by pigments (melanin, hemoglobin), water, proteins, collagen fibers and other structural components of the organism. Absorption of the laser beam from the tissue target is the essential and primary advantage of their use..$^{2,3}$

Development of laser devices in a wide range of medical specialties such as Ophthalmology, ENT, Dermatology, Gynecology, Urology, Dentistry and Surgery is impressive. Numerous laser types are also applied in a variety of Oral and Maxillofacial surgical interventions, with different indications and features. ${ }^{4}$

Sinus lift elevation using bone grafts is a popular, scientifically documented method of bone augmentation, in cases with residual bone background of the area for implant placement. This surgical technique is characterized as a relatively extensive surgical procedure, with adequate postoperative morbidity of the patient, especially in cases that is performed by classical scalpel technique. ${ }^{5}$ One of the most severe intra-operative complications demanding immediate action when mechanical rotary tools are used is severe and vigorous bleeding due to dehiscence of vascular structures located within the area of osteotomy. ${ }^{6}$

The purpose of this paper is to highlight the possibility of integrating laser devices within the abovementioned surgical technique, in order to perform as atraumatic and uncomplicated intraoral procedures as possible as. Specifically, we describe a patient that underwent open sinus lift surgery for placement of bone graft, with the aid of Er, Cr: YSGG laser device. In discussion, advantages and disadvantages of applying lasers to perform intraoral surgical interventions are highlighted, differences in potential postoperative complications compared to classical scalpel technique are discussed and special characteristics that led to the selection of Er, Cr: YSGG laser device are described.

\section{Case report}

A 47 years old male was referred for a fixed implant-supported restoration on the upper left half of the maxilla. Patient's medical history was significant for hypothyroidism, controlled with a daily per os levothyroxine pill medication.

After clinical evaluation, analysis of panoramic radiography and CT dental scan, the presence of poor bone base for implant placement was noted (Figure 1).

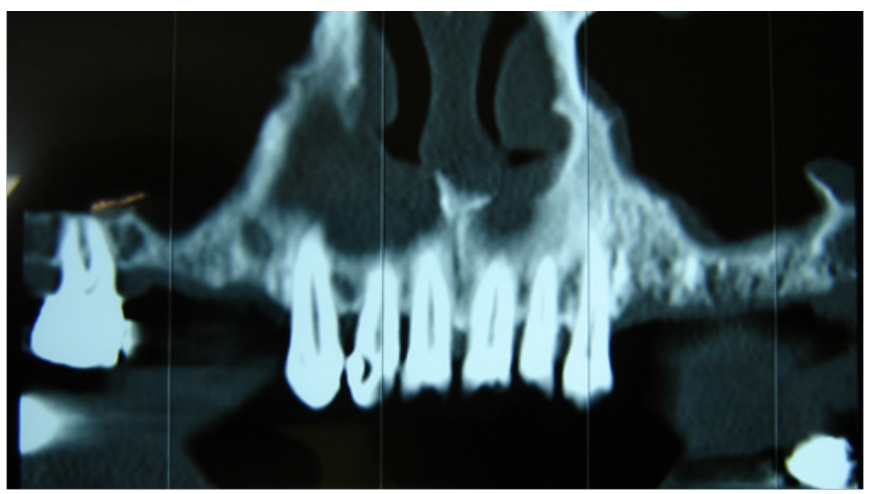

Figure I Initial panoramic radiograph of the maxilla with extended bone loss observed on the left side. 
Bone augmentation through laser-assisted open sinus lift technique, simultaneously with placement of four endosseous implants, to restore the edentulous area and achieve adequate aesthetic and functional dental arch was recommended. The patient became aware about chances of success, possible complications of surgery and consented with the proposed treatment regimen, summarized below:

Under block anesthesia of infraorbital, posterior upper alveolar and major palatal nerve, as well as local anesthetic infiltration of the premolars and molars in the left half of maxilla, a horizontal mucosal incision on the alveolar crest was made using Er, Cr: YSGG Laser (Biolase, Waterlase), followed by two diverging releasing sections. Detachment of trapezoidal, full-thickness flap and exposure of the lateral sinus wall followed. Using Er, Cr: YSGG laser device, a bone fragment of $1 \mathrm{~cm}$ height and $2 \mathrm{~cm}$ width, on the lateral sinus wall took place (Figure 2). According to preoperative calculations, the lower side of the bone fragment was $4 \mathrm{~mm}$ above the alveolar ridge, in order to achieve access into the sinus cavity. Detachment of the bone fragment was accomplished using relatively blunt chisel and transpositioned on the roof of the space where the graft would be placed (Figure 3). After a complete, uncomplicated detachment of Schneiderian membrane, 2grams of coarse-grained, bovine graft (BioOSS, Geistlich) were placed through the bone window and packed. Simultaneous positioning of four osseointegrated implants, under the guidance of a surgical splint, according to preoperative calculations, since the height of the alveolar ridge was sufficient to guarantee initial stability was accomplished (Figure 4). Finally an absorbable collagenous bilayer membrane (Bio-Gide, Geistlich) was placed in the lateral wall of the sinus and the flap was fixed in place with 3-0 silk suture. Postoperatively the patient was administered amoxicillin $1 \mathrm{gm}$ every 8 hours, painkillers and nasal decongestants. No postoperative complication was noted whereas postoperative radiographic and clinical course was considered fully satisfactory (Figure 5).

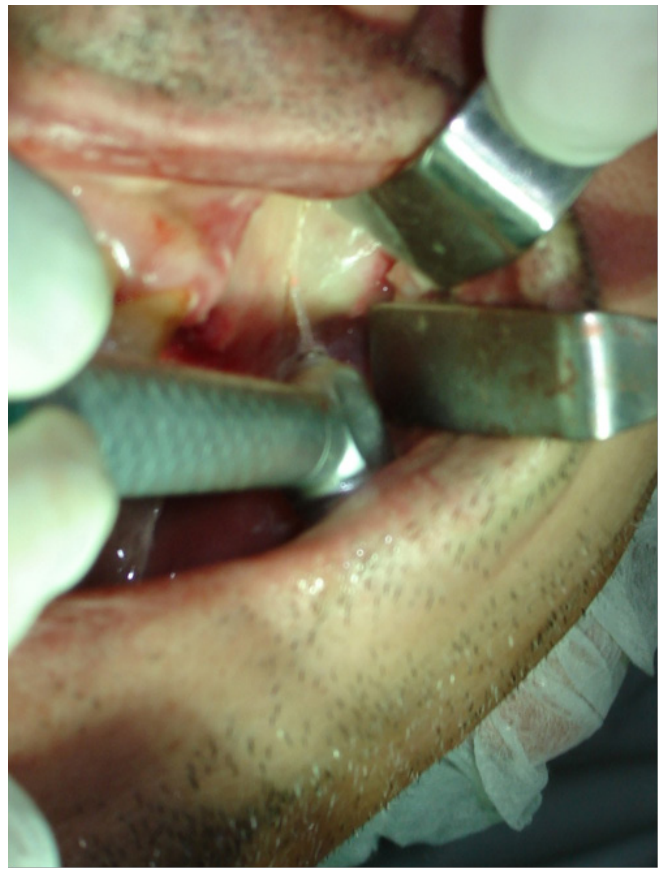

Figure 2 Bone fragment on the lateral sinus wall carried out using $\mathrm{Er}, \mathrm{Cr}$ : YSGG laser.

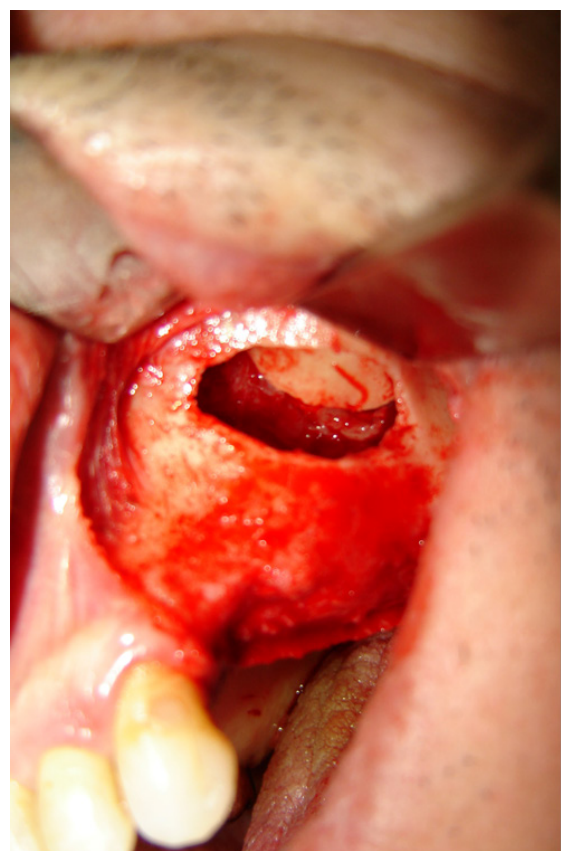

Figure 3 Detachment of the bone fragment accomplished. Bone tissue transpositioned on the roof of the graft space.

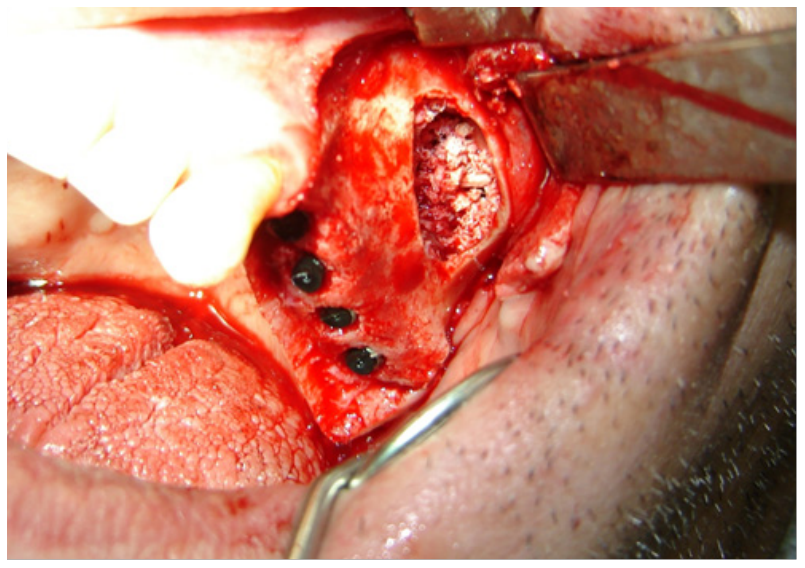

Figure 4 Four implants inserted on the edentulous alveolar ridge. Sinus fulfilled with bone graft.

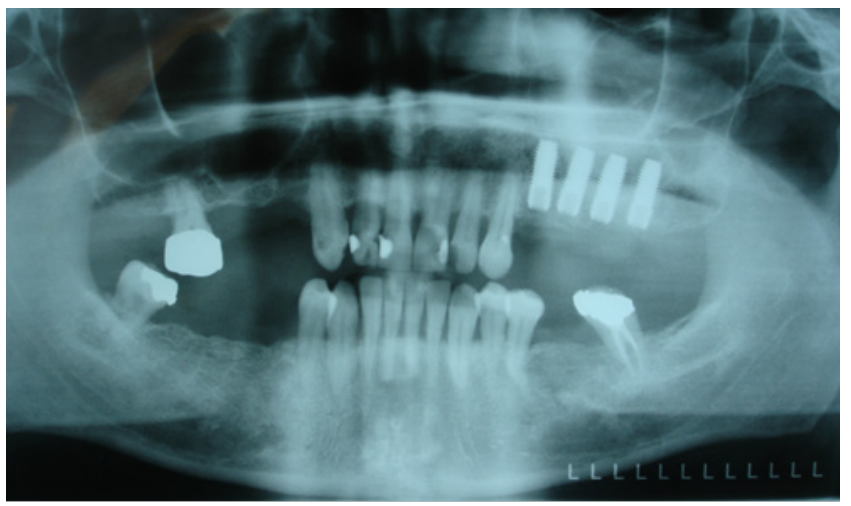

Figure 5 Final panoramic radiograph showing inserted implants and sinus lift. 


\section{Discussion}

Usage of laser devices in oral and maxillofacial surgery allows performing relatively mild-atraumatic interventions.

Among the main advantages, hemostatic effect is mentioned, achieved with degeneration and dehydration of proteins and collagen of the vascular wall, leading to concomitant thrombosis of small blood and lymph vessels. Hemostasis is judged as sufficient to develop a clean, 'dry' surgical field, facilitating intraoperative manipulations. Furthermore, the postoperative hematoma is less likely to occur, while the possibility of carrying out surgical procedures in patients without interruption or modification on anticoagulant therapy is extended, as well as in patients with blood coagulation disorders. Particularly, in the field of sinus surgery, development of bleeding from a large vessel (posterior superior alveolar and infraorbital artery) reinforces the use of such devices that more effectively manage such complications. ${ }^{7,8}$

Laser devices with potential use in soft and hard tissues belong to Er, Cr: YSGG, Er: YAG and Nd: YAG categories. Selection of Erbium laser gives the big advantage of developing less thermal necrosis to surrounding tissues through water spray usage, a particularly important feature for maintaining bone and sinus membrane integrity. The hard tissue cutting mechanism in the Erbium laser family is due to the interaction of the emitted energy with water spray, identified as "hydrokinetic effect". The fact that the absorption of laser energy by water leads to violent explosions, responsible for the removal of calcified hard tissues such as bone and tooth structure without causing damage to surrounding tissues, led us to the adoption of the specific laser for carrying out the aforementioned procedure. ${ }^{9}$

Open sinus lift surgery with the aid of a laser device is a notable alternative technique since it achieves minimal wound development in a relatively extensive surgical procedure and reduced postoperative morbidity of the patient. It is now assumed that laser applications achieve reduced postoperative pain and swelling, making their use very attractive. ${ }^{10}$

Also, bone cutting using Er, Cr: YSGG laser reduces surgical time, vibrations and noise sounds compared to conventional rotary hand pieces, eliminating surgical stress. ${ }^{11}$

The bactericidal action of the laser beam leads to bacteremia reduction, observed in conventional scalpel surgery, while it reduces the possibility of wound contamination. Moreover, this fact increases safety in high risk patients-diabetes, valvular diseases. Finally, local eradication of bacteria is of particular importance when adding bone graft, as in our patient, because it requires aseptic conditions. ${ }^{3}$

Additionally surgeon's maneuver accuracy is increased by the guidance of spot laser beam, resulting in more atraumatic technique. ${ }^{2}$

Of course we must not consider the use of laser devices panacea. There are disadvantages associated with high cost of equipment and special training of the doctor for proper use and configuration of the device parameters (pulse frequency, power intensity). ${ }^{4}$

Given the lack of touch sense during laser-assisted sinus lift, special attention during the cutting of the bone window and knowledge of cutting depth is required, because perforation of Schneider's membrane is probable. The latter is a complication that could lead to treatment failure, while longer-term consequences such as nasal drainage weakness of the damaged respiratory type epithelium is equally important. For this reason, the laser handle must be constantly, in a stable slow motion, since sinus lateral wall is thin and as sinus membrane is approached, the distance between tip and target tissue should increase. ${ }^{12}$
In addition, compressed air of the laser handle must be avoided to enter detached flaps, with appropriate manipulations, to avoid air embolism.

Finally we should not escape the risk of eye damage if laser beam comes in contact with them (cornea or retina burns, lens opacity), therefore prophylactic protective glasses for the medical team and the patient are considered imperative. ${ }^{8}$

In conclusion, we can assume that $\mathrm{Er}, \mathrm{Cr}$ : YSGG laser is a valuable tool in open sinus lift surgery, if used at recommended settings mode and doctor possesses appropriate medical training. The risk of perforation of the Schneiderian membrane is less compared to conventional rotary tools, allows comfortable handling during surgery, offers less invasive interventions and achieves reduced operative time, postoperative morbidity and complications.

\section{Funding}

None.

\section{Acknowledgements}

None.

\section{Conflicts of interest}

Authors declare that there is no conflict of interest.

\section{References}

1. Kaufman E. Maxillary Sinus Elevation: An Overview. J Esthet Restor Dent. 2003;15(5):272-282.

2. Stubinger S. Advances in bone surgery: the Er: YAG laser in oral surgery and implant dentistry. Clin Cosmet Investig Dent. 2010;30(2):47-62.

3. Deppe H, Horch HH. Laser applications in oral surgery and implant dentistry. Lasers Med Sci. 2007;22(4):217-221.

4. Fasbinder DJ. Dental laser technology. Compend Contin Educ Dent. 2008;29(8):452-454.

5. Wang X, Zhang C, Matsumoto K. In vivo study of the healing processes that occur in the jaws of rabbits following perforation by an Er, Cr: YSGG laser. Lasers Med Sci. 2005;20(1):21-27.

6. Valente NA. Anatomical considerations on the alveolar antral artery as related to the sinus augmentation surgical procedure. Clin Implant Dent Relat Res. 2015.

7. Solar P, Geyerhofer U, Traxler H, et al. Blood supply to the maxillary sinus relevant to sinus floor elevation procedures. Clin Oral Implants Res. 1999;10(1):34-44.

8. Stern A, Green J. Sinus lift procedures: an overview of current techniques. Dent Clin North Am. 2012;56(1):219-233.

9. Sohn DS, Lee JS, An KM, et al. Erbium, chromium: yttrium-scandiumgallium-garnet laser-assisted sinus graft procedure. Lasers Med Sci. 2009;24(4):673-677.

10. Colucci V, do Amaral FL, Pecora JD, et al. Water flow on erbium: yttriumaluminum-garnet laser irradiation: effects on dental tissues. Lasers Med Sci. 2009;24(5):811-818.

11. Mercer C. Lasers in dentistry: a review. Part 1. Dent Update. 1996;23(2):74-80.

12. Maiman TH. Stimulated optical radiation in ruby. Nature. 1960;187:493494. 